\title{
Construcción y propiedades psicométricas de un cuestionario para medir el nivel de adicción a videojuegos en red (CUAVIR) de la ciudad de Juliaca - 2020
}

\section{Construction and psychometric properties of a questionnaire to measure the level of addiction to online video games (CUAVIR) in the city of Juliaca - 2020}

\author{
Yeni Bellido Mamani ${ }^{1}$, Monica Cahuana Cuti ${ }^{2}$
}

\begin{abstract}
RESUMEN
Objetivo: Construir y determinar las propiedades psicométricas de un cuestionario para medir el nivel de adicción a videojuegos en red, en adolescentes y jóvenes cuyas edades oscilan los 12 a 25 años. Metodología: Diseño no experimental, tipo estudio psicométrico, con corte transversal y de enfoque cuantitativo. La muestra está conformada por 145 sujetos que participaron voluntariamente del estudio a partir de una aplicación ambulatoria, el instrumento construido denominado "CUAVIR", consta de 42 ítems distribuidos en 5 dimensiones (Abstinencia, Resistencia, Interferencia con Actividades, Dependencia y Conciencia de Enfermedad). Para la validez de contenido se aplicó el criterio de jueces expertos, los resultados se analizaron a través de la Prueba Binomial, el coeficiente de validez se midió a través de $\mathrm{V}$ de Aiken, para la medición del grado de consistencia interna se utilizó el coeficiente Alfa de Cronbach. Resultados: A la prueba Binominal se determinó que las dimensiones e ítems son adecuados y significativos, con respecto a la validez de contenido el $V$ de Aiken arrojó intervalos de confianza de 0.89 para los ítems, lo que indica que el cuestionario posee un alto grado de validez en cuatro esenciales criterios (Congruencia, Claridad, Dominio del Constructo y Contexto). Con respecto a la consistencia interna, el coeficiente Alfa de Cronbach mostró que las dimensiones obtuvieron valores de 0.942 lo que demuestra que el instrumento es confiable. El análisis factorial confirmatorio evidenció que el instrumento posee características que permiten el procesamiento por el (AFC) y la varianza total explicada mostró que con cinco factores la varianza total acumulada logra explicarse un 52,358 \% lo que indicaría que es apropiado el trabajo con cinco dimensiones. Conclusión: El cuestionario para medir el nivel de adicción a videojuegos en red (CUAVIR) evidencia buena consistencia interna y validez de constructo.
\end{abstract}

Palabras clave: Adicción; videojuegos en red; abstinencia; resistencia; interferencia con actividades; dependencia y conciencia de enfermedad.

\begin{abstract}
Objective: To construct and determine the psychometric properties of a questionnaire to measure the level of addiction to online video games, in adolescents and young people whose ages range from 12 to 25 years. Methodology: Non-experimental design, type psychometric study, with a cross section and quantitative approach. The sample is made up of 145 subjects who voluntarily participated in the study from an outpatient application, the instrument constructed called "CUAVIR", consists of 42 items distributed in 5 dimensions (Abstinence, Resistance, Interference with Activities, Dependence and Awareness of Illness). For content validity, the criterion of expert judges was applied, the results were analyzed through the Binomial Test, the validity coefficient was measured through Aiken's $V$, and the alpha
\end{abstract}

${ }^{1}$ Universidad Peruana Unión, Juliaca, Perú.

Orcid ID: 0000-0002-3505-5381

¿Universidad Peruana Unión, Juliaca, Perú.

Orcid ID: 0000-0001-9069-1068 
coefficient was used to measure the degree of internal consistency of Cronbach. Results: The Binominal test determined that the dimensions and items are adequate and significant, with respect to content validity, Aiken's $V$ yielded confidence intervals of 0.89 for the items, which indicates that the questionnaire has a high degree validity in four essential criteria (Congruence, Clarity, Domain of the Construct and Context). Regarding internal consistency, Cronbach's alpha coefficient showed that dissensions obtained values of 0.942 , which shows that the instrument is reliable. The confirmatory factor analysis showed that the instrument has characteristics that allow processing by the (CFA) and the total variance explained showed that with five factors the total accumulated variance can be explained by $52.358 \%$, which would indicate that the work with five dimensions is appropriate. Conclusion: the questionnaire to measure the level of addiction to online video games (CUAVIR) shows good internal consistency and construct validity.

Keywords: Addiction; network video games; abstinence; resistance; interference with activities; dependence and disease awareness.

\section{INTRODUCCIÓN}

Hoy en día la adicción a los videojuegos por lo regular afecta especialmente a niños adolescentes y jóvenes a nivel mundial, ya que están al alcance de todos como; portátiles, tabletas, computadoras y hasta en los celulares, que son objetos de uso diario, que, utilizados de manera errónea en una etapa temprana de desarrollo pueden ser causa del deterioro temprano y tardío del desarrollo cognitivo y psicosocial. Carbonell (2019) menciona que el trastorno de juego por internet, conocido como "adicción a videojuegos", se caracteriza por un patrón de comportamiento de juego persistente y recurrente que conlleva un deterioro o malestar clínicamente significativo. Luque y Degoy (2012) indican que existe un porcentaje considerable en que los videojuegos reducen el tiempo de actividades académicas y profesionales, y esto genera problemas con la familia o pareja.

La Organización Mundial de la Salud (2010) menciona que la adicción es una enfermedad física y psicoemocional que crea una dependencia hacia una sustancia, actividad o relación. El diario el País (2017) informa que Organización Mundial de la Salud (OMS) incluirá, por primera vez, el trastorno por videojuegos como enfermedad mental, al incluirlo en su próxima edición de la Clasificación Internacional de Enfermedades (ICD-11), que no se actualiza desde 1990, denominándola como un tipo de juegos que comparten mecanismos etiológicos con las adicciones y pueden producir una enfermedad el "juego patológico" el Diario correo (2018) reporta un estudio el cual revela que el $45 \%$ de los videogamers en el Perú son mujeres, la investigación también precisa que el $60 \%$ de los consumidores de videojuegos electrónicos son adultos jóvenes, en cuanto a los rangos de edad, el $60 \%$ de los jugadores son personas que oscilan entre los 18 a 35 años de edad. Esto indica que la mayoría de los gamers no son niños ni adolescentes, sino adultos jóvenes.

Por su parte Echeburúa y Corral (2009) consideran que el aspecto adictivo de una conducta no se debe tanto a la frecuencia con que se realiza, sino al tipo de relación que se establece con ella. Los videojuegos tienen una sensación de satisfacción, por lo que deben promover su reutilización. Son divertidos, ágiles, cambiantes, atractivos y desafiantes, fáciles de usar, pero no tan fáciles de dominar, otorgan premios y castigos, convirtiéndose en motivación para la acción, esto motiva a los consumidores a seguir jugando reiteradas veces en distintos horarios llegando a perder la noción del tiempo.

Goldberg (1995) publicó en su foro una parodia del DSM y se inventó un nuevo trastorno, el Síndrome de Adicción a Internet. Young (1996) presentó informacion de nternet addiction: The emergence of a new disorder en el congreso de la American Psychological Association, celebrado en Toronto, desde entonces el tema ha sido ampliamente discutido en los medios de comunicación y en la literatura científica. Carbonell, Guardiola, Beranuy y Bellés (2009) señalan que el interés por la posible adicción a los videojuegos, juegos de rol online, televisión y teléfonos móviles ha dado lugar a lo que Griffiths (2005) denominó "adicciones tecnológicas".

Por ello el estudio de este constructo se ha incrementado al igual que la construcción de instrumentos para su medición; donde Lemmens y Valkenburg (2009) desarrollan y validan la escala de Adicción a videojuegos para adolescentes, por 
dos razones: ellos juegan estos juegos más que los adultos y porque están en la etapa vulnerable a las adicciones a juegos en línea. Cuenta, con una versión de 21 ítems y una abreviada de 7 ítems, aplicada en Europa, (Huaraya 2013) realizo una investigación en la ciudad de Arequipa dicho instrumento consta de 7 dimensiones: (1) predominancia (2) tolerancia (3) modificación del estado de ánimo (4) abstinencia (5) recaída (6) conflicto (7) problemas.

De igual modo, Choliz y Marco (2010) crearon el cuestionario test de dependencia de videojuegos (TDV) adaptada posteriormente en el año 2017, usando los criterios del DSMIVTR (American Psychiatric Association, 2000), con 25 ítems que es de tipo escala likert que contiene 4 factores: Abstinencia, Abuso y Tolerancia, Problemas ocasionados por los videojuegos y Dificultad en el control.

Ante esta realidad, ha surgido una inquietud frente a la investigación sobre el constructo de adicción a los videojuegos, pues los estudios demuestran que existen cambios neuroquímicos involucrados en las personas con desórdenes adictivos y que además existe predisposición biogenética a desarrollar esta enfermedad. Sin embargo, la adicción es una enfermedad tratable y la recuperación es posible, a diferencia que en los casos de gravedad y deterioro generalizado esto es irreversible. Por lo tanto, es necesario poder identificar el grado de adicción para así poder llevar un tratamiento a tiempo. Por otro lado, muchos de los estudios revisados muestran instrumentos que fueron adaptados en otras poblaciones, pues existe un mínimo porcentaje adaptado a nuestra cultura. Es por ello que el objetivo del presente trabajo es crear un instrumento psicológico que nos permita medir el nivel de adicciones en adolescentes y jóvenes.

\section{METODOLOGÍA}

Esta investigación empírica es de diseño no experimental, ya que el investigador no controla, ni manipula las variables del estudio; así mismo es de corte transversal, (Hernández, Fernández, y Baptista, 2014). Del mismo modo, el presente trabajo es de tipo cuantitativo, puesto que se iniciará con una idea que se delimita y de la que se obtendrán objetivos y se investigan diversas fuentes para construir un marco teórico. (Hernández, et. 2014). En este caso se construirá un instrumento y quedarán determinadas sus propiedades psicométricas.

Montero y León (2007) clasifican este tipo de investigación como estudios instrumentales puesto que su objetivo es desarrollar un instrumento desde la elaboración hasta la determinación de las propiedades psicométricas.

\section{Participantes}

La investigación se ejecutó en la provincia de San Román - Juliaca, con una población ambulatoria de diferentes cabinas públicas de internet, con edades que oscilan entre 12 y 25 años de edad. La muestra está constituida por 145 personas elegidas ambulatoriamente, quienes luego del consentimiento informado, fueron evaluadas con el "cuestionario que mide nivel de adicción a videojuegos en red (CUAVIR)", dicha población tiene como características de sexo femenino y masculino, con un grado de instrucción ya sean universitarios y estudiantes de secundaria.

\section{Proceso de construcción}

El cuestionario de Adicción a Videojuegos en red, fue construido con 42 ítems, donde cada ítem, es valorado en una escala tipo Likert de cinco puntos que va desde uno (nunca) hasta cinco (siempre). Se construyó bajo el enfoque del psiquiatra Ivan K. Goldberg, modificada por Young en 1996, tomando como referencia las dimensiones abstinencia, resistencia, interferencias con actividades, dependencia, conciencia de enfermedad.

- Dimensión Abstinencia: Es el malestar emocional cuando se interrumpe el juego o cuando se lleva un tiempo sin jugar. Ítems 1 , 26, 30, 32, 33, 40, 42.

- Dimensión de Resistencia: Es la necesidad del juego, no se puede dejar de jugar, ya que este le provoca sentimientos de placer. Ítems $3,5,7,8,10,11,12$.

- Dimensión de Interferencia con actividades: Es la alteración o perturbación del desarrollo normal de una cosa mediante la interposición de otra que puede llegar a resultar un 
obstáculo. Ítems $15,18,19,21,22,23,25$, $27,28,29,31,34,35,36,37,38,39,41$.

- Dimensión de Dependencia: es la priorización del juego sobre todas las actividades extracurriculares. Ítems 6, 9, 13, 14, 16, 17, 20.

- Conciencia de enfermedad: Se refiere al conocimiento claro del grado de adicción en el que se encuentra, donde la persona busca ayuda para instaurar un cambio integral en su vida. Ítems 2,4.

Una vez construido el instrumento con 42 ítems, se procedió a la validez de contenido mediante el criterio de expertos. El grupo de expertos estuvo conformado por: psicólogos especializados en la docencia universitaria, adicciones, investigación, metodología, clínica y lingüista. Los expertos evaluaron cada uno de los ítems teniendo en cuenta la claridad, congruencia, contextos y dominio de cada uno de ellos, así como, a la dimensión que pertenecían. Luego se realizaron las modificaciones sugeridas y se llevó a cabo la aplicación del instrumento con 145 personas. Se construyó la base de datos en el programa estadísticos SPSS 24.0. Los índices de confiabilidad (Alfa de Cronbach $=0.942$ ) indican que la prueba es muy confiable y su validez ( $V$ de Aiken $=0.89$ ) indica que se trata de un instrumento que mide lo que verdaderamente pretende medir (Hernandez, Roberto; Fernández, Collado; Baptista, 2010).

\section{RESULTADOS}

\section{Validez de contenido por juicio de expertos}

La validez de contenido se obtuvo mediante el criterio de expertos en el área, todos ellos con reconocida trayectoria académica de nivel universitario, posgrado e investigadores. Así mismo Escurra (1988) menciona que los reactivos cuyos valores sean mayores o iguales a 0.80 se consideran válidos para la prueba. De esta manera se procedió a calcular los coeficientes mediante el método de validez $\mathrm{V}$ de Aiken para el test, siendo esta unidimensional.

En la tabla 1 se muestra la validez de $V$ de Aiken por jueces donde se obtuvo un puntaje de 0,92 en cuanto a claridad, un 0.94 en congruencia, un 0.75 en contexto y un 0.93 en dominio del constructo, obteniendo una media de 0.89 para todo el instrumento lo que indica que el instrumento posee un alto grado de validez.

Tabla 1

Validez por $V$ de Aiken por dimensiones

\begin{tabular}{llllll} 
Variable & Claridad & Congruencia & Contexto & $\begin{array}{c}\text { Dominio del } \\
\text { constructo }\end{array}$ & Total \\
\hline Adicción a videojuegos & & & & & \\
Abstinencia & 0.81 & 1 & 0.75 & 0.97 & 0.88 \\
Resistencia & 0.96 & 0.75 & 0.75 & 0.75 & 0.80 \\
Interferencia de Actividades & 0.91 & 0.96 & 0.75 & 0.97 & 0.90 \\
Dependencia & 1 & 0.96 & 0.75 & 0.90 & 0.90 \\
Conciencia De Enfermedad & 0.95 & 1 & 0.75 & 1 & 0.93 \\
Total & 0.92 & 0.94 & 0.75 & 0.93 & 0.89 \\
\hline
\end{tabular}

\section{Validez del constructo}

En la tabla 2 se muestra la puntación de la medida de Kaiser Meyer Olkin obteniendo un 0,874 con un Sig. $<0.05$ lo que indicaría que el instrumento guarda características para ser procesado por el análisis factorial exploratorio. 
Tabla 2

Prueba de MKO y Bartlett

\begin{tabular}{llc} 
Medida Kaiser-Meyer-Olkin de adecuación de muestreo &, 874 \\
Prueba de esfericidad de Bartlett & Aprox. Chi-cuadrado & 3327,293 \\
& GI & 861 \\
\hline
\end{tabular}

En la tabla 3 se observa la varianza total explicada, donde se obtiene 5 factores correlacionados por determinadas características en común, sin embargo, se debe considerar la cantidad de ítems pertenecientes a cada factor, para ser denominada como dimensión, entonces se obtiene como primeros resultados máximo a la varianza acumulada del factor siendo explicado con el 52,358 \% del total, lo que confirma el uso de 5 factores en el instrumento.

Tabla 3

Varianza total explicada

\begin{tabular}{|c|c|c|c|c|c|c|c|c|c|}
\hline \multirow{2}{*}{$\begin{array}{l}\text { Compo- } \\
\text { nente }\end{array}$} & \multicolumn{3}{|c|}{ Autovalores iniciales } & \multicolumn{3}{|c|}{$\begin{array}{l}\text { Sumas de cargas al cuadrado } \\
\text { de la extracción }\end{array}$} & \multicolumn{3}{|c|}{$\begin{array}{l}\text { Sumas de cargas al cuadrado de la } \\
\text { rotación }\end{array}$} \\
\hline & Total & $\begin{array}{c}\% \text { de } \\
\text { varianza }\end{array}$ & $\begin{array}{l}\% \text { acumu- } \\
\text { lado }\end{array}$ & Total & $\begin{array}{c}\% \text { de } \\
\text { varianza }\end{array}$ & $\begin{array}{l}\% \text { acu- } \\
\text { mulado }\end{array}$ & Total & $\begin{array}{l}\% \text { de va- } \\
\text { rianza }\end{array}$ & $\begin{array}{l}\% \text { acumu- } \\
\text { lado }\end{array}$ \\
\hline 1 & 13,670 & 32,548 & 32,548 & 13,670 & 32,548 & 32,548 & 8,716 & 20,752 & 20,752 \\
\hline 2 & 3,027 & 7,207 & 39,756 & 3,027 & 7,207 & 39,756 & 4,429 & 10,546 & 31,298 \\
\hline 3 & 1,989 & 4,735 & 44,490 & 1,989 & 4,735 & 44,490 & 3,546 & 8,444 & 39,742 \\
\hline 4 & 1,748 & 4,163 & 48,653 & 1,748 & 4,163 & 48,653 & 3,382 & 8,052 & 47,794 \\
\hline 5 & 1,556 & 3,705 & 52,358 & 1,556 & 3,705 & 52,358 & 1,917 & 4,563 & 52,358 \\
\hline 6 & 1,356 & 3,228 & 55,586 & & & & & & \\
\hline 7 & 1,194 & 2,843 & 58,429 & & & & & & \\
\hline 8 & 1,177 & 2,802 & 61,230 & & & & & & \\
\hline 9 & 1,123 & 2,674 & 63,904 & & & & & & \\
\hline 10 & 1,058 & 2,519 & 66,423 & & & & & & \\
\hline 11 & 1,023 & 2,435 & 68,858 & & & & & & \\
\hline 12 & ,942 & 2,244 & 71,102 & & & & & & \\
\hline 13 & ,883 & 2,102 & 73,204 & & & & & & \\
\hline 14 & ,786 & 1,870 & 75,074 & & & & & & \\
\hline 15 & ,748 & 1,781 & 76,856 & & & & & & \\
\hline 16 & ,708 & 1,686 & 78,542 & & & & & & \\
\hline 17 & ,668 & 1,591 & 80,133 & & & & & & \\
\hline 18 & ,606 & 1,444 & 81,577 & & & & & & \\
\hline 19 &, 579 & 1,378 & 82,955 & & & & & & \\
\hline 20 &, 562 & 1,339 & 84,294 & & & & & & \\
\hline 21 &, 522 & 1,243 & 85,537 & & & & & & \\
\hline 22 & ,502 & 1,195 & 86,732 & & & & & & \\
\hline 23 & ,490 & 1,168 & 87,899 & & & & & & \\
\hline 24 & ,475 & 1,132 & 89,031 & & & & & & \\
\hline 25 & ,429 & 1,021 & 90,053 & & & & & & \\
\hline 26 & ,412 & ,981 & 91,034 & & & & & & \\
\hline 27 & ,410 & ,977 & 92,011 & & & & & & \\
\hline 28 & ,376 & ,895 & 92,906 & & & & & & \\
\hline
\end{tabular}




\begin{tabular}{llll|}
29 &, 341 &, 813 & 93,719 \\
30 &, 327 &, 779 & 94,498 \\
31 &, 292 &, 695 & 95,193 \\
32 &, 259 &, 617 & 95,810 \\
33 &, 238 &, 568 & 96,377 \\
34 &, 224 &, 533 & 96,910 \\
35 &, 211 &, 503 & 97,413 \\
36 &, 207 &, 492 & 97,905 \\
37 &, 181 &, 431 & 98,335 \\
38 &, 168 &, 400 & 98,735 \\
39 &, 165 &, 392 & 99,127 \\
40 &, 152 &, 362 & 99,489 \\
41 &, 129 &, 308 & 99,797 \\
42 &, 085 &, 203 & 100,000 \\
\hline
\end{tabular}

Método de extracción: análisis de componentes principales

En la tabla 4, se observa la matriz rotada para el cuestionario en estudio, lo que indica que los ítems 15 , $18,19,21,22,23,25,27,28,29,31,34,35,36,37,38,39,41$, pertenecen al primer factor denominado interferencia de actividades, en cuanto a los segundos factores con los ítems $6,9,13,14,16,17,20$, 24, denominado dependencia, de la misma forma los ítems 1, 26, 30, 32, 33, 40, 42, el tercer factor denominado abstinencia, el cuarto factor denominado resistencia con los ítems $3,5,7,8,10,11,12$, siendo el último factor denominado conciencia de enfermedad con los ítems 2,4.

Tabla 4

Matriz de componente rotado para CUAVIR

\begin{tabular}{|c|c|c|c|c|c|}
\hline \multicolumn{6}{|c|}{ Matriz de componente rotado ${ }^{a}$} \\
\hline & \multicolumn{5}{|l|}{ Componente } \\
\hline & Interferencia con actividades & Dependencia & $\begin{array}{l}\text { Abstinen- } \\
\text { cia }\end{array}$ & Resistencia & $\begin{array}{l}\text { Conciencia de enfer- } \\
\text { medad }\end{array}$ \\
\hline P41 &, 779 & & & & \\
\hline P39 & ,776 & & & & \\
\hline P27 & ,708 & & & & \\
\hline P37 & ,695 & & & & \\
\hline P28 & ,688 & & & & \\
\hline P25 & ,684 & & & & \\
\hline P29 & ,639 & & & & \\
\hline P31 & ,626 & & & & \\
\hline P36 &, 593 & & & & \\
\hline P35 &, 589 & & & & \\
\hline P22 &, 587 & & & & \\
\hline P23 &, 587 & & & & \\
\hline P38 &, 543 & & & & \\
\hline P21 &, 537 & & & & \\
\hline P34 &, 519 & & & & \\
\hline P18 & ,496 & & & & \\
\hline P15 & ,452 & & & & \\
\hline P19 & ,415 & & & & \\
\hline P16 & & ,748 & & & \\
\hline
\end{tabular}




\begin{tabular}{|c|c|c|c|c|}
\hline P20 & ,661 & & & \\
\hline P6 & ,654 & & & \\
\hline P13 & ,555 & & & \\
\hline P24 &, 527 & & & \\
\hline P17 & ,508 & & & \\
\hline P9 & ,487 & & & \\
\hline P14 & ,440 & & & \\
\hline P40 & & ,617 & & \\
\hline P30 & & 606 & & \\
\hline P42 & & ,578 & & \\
\hline P33 & & ,569 & & \\
\hline P26 & & ,547 & & \\
\hline P1 & & ,539 & & \\
\hline P32 & & ,386 & & \\
\hline P7 & & & ,596 & \\
\hline P8 & & & ,595 & \\
\hline P5 & & & ,573 & \\
\hline P10 & & & ,565 & \\
\hline P3 & & & ,563 & \\
\hline P12 & & & 383 & \\
\hline P11 & & & ,382 & \\
\hline P2 & & & & 628 \\
\hline P4 & & & & ,574 \\
\hline
\end{tabular}

Fuente: elaboración propia.

Método de extracción: análisis de componentes principales.

Método de rotación: Varimax con normalización Kaiser.

a. La rotación ha convergido en 10 iteraciones.

\section{Confiabilidad por alfa de Cronbach}

En la tabla 5, se observa el Alfa de Cronbrach de cada dimensión donde abstinencia obtiene un puntaje de 0.663 la cual demuestra una confiabilidad aceptable, en cuanto a resistencia con hay un puntaje de 0.593 demostrando una confiabilidad media o moderada, mientras la dimensión de interferencia obtuvo un puntaje de 0.914 y la dimensión dependencia, obtuvo un puntaje de 0.794 demuestran una confiabilidad aceptable, así mismo la dimensión conciencia de enfermedad, obtuvimos un puntaje de 0.825 y un total de las cinco dimensiones con 42 ítems, obteniendo un puntaje de 0.942 demuestran una confiabilidad aceptable.

Tabla 5

Estadísticos de Confiabilidad por Alfa de Cronbach por dimensiones

\begin{tabular}{lll} 
Variable & Alfa de Cronbach & Nivel de confianza \\
Adicciones a videojuegos en red &, 942 & Alta confianza \\
Abstinencia &, 663 & Alta confianza \\
Resistencia &, 593 & Moderada confianza \\
Interferencia de actividades &, 914 & Alta confianza \\
Dependencia &, 794 & Alta confianza \\
Conciencia de enfermedad &, 825 & Alta confianza \\
\hline
\end{tabular}


Tabla 6

Baremos

\begin{tabular}{|c|c|c|c|c|c|c|c|}
\hline \multicolumn{4}{|c|}{ PERCENTIL } & \multicolumn{2}{|c|}{ CATEGORÍA } & & \\
\hline $1-29$ & & & BAJO & & & & \\
\hline $30-74$ & & & MEDIO & & & & \\
\hline $75-100$ & & & ALTO & & & & \\
\hline & Iterferencia & Dependencia & Abstinencia & Resistencia & Consciencia & General & PC \\
\hline Alto & 44 a más & 19 a más & 18 a más & 20 a más & 4 a más & 106 a más & $75-99$ \\
\hline Medio & $24-43$ & $12-18$ & $10-17$ & $14-19$ & 3 & $63-105$ & $30-70$ \\
\hline Bajo & $0-23$ & $0-11$ & $0-9$ & $0-13$ & $0-2$ & $0-62$ & $1-25$ \\
\hline$x$ & 35.9 & 15.9 & 14.3 & 17 & 3.3 & 86,6 & - \\
\hline D.S & 13.9 & 6,3 & 6.1 & 5.5 & 1.6 & 27 & - \\
\hline
\end{tabular}

Tabla 7

Ítems de cuestionario CUAVIR

\begin{tabular}{|c|c|c|c|c|c|}
\hline ITEM & CUAVIR & $\mathbf{N}$ & RV & AV & AM \\
\hline 01 & Prefiero jugar un videojuego en vez de estar en clases. & & & & \\
\hline 02 & $\begin{array}{l}\text { He faltado seguidamente a clases por estar jugando videojuegos } \\
\text { en red. }\end{array}$ & & & & \\
\hline 03 & Juego más de una hora al día. & & & & \\
\hline 04 & $\begin{array}{l}\text { Pienso que exageran todos al decirme que soy adicto a los video- } \\
\text { juegos. }\end{array}$ & & & & \\
\hline 05 & $\begin{array}{l}\text { Cuando yo me quedo solo en casa, no encuentro otra actividad } \\
\text { interesante que el estar jugando con la computadora. }\end{array}$ & & & & \\
\hline 06 & Prefiero jugar que conversar con una persona del sexo opuesto. & & & & \\
\hline 07 & Cuando menos lo espero siento que anochece rápidamente. & & & & \\
\hline 08 & Mientras juego no siento que paso más de dos horas. & & & & \\
\hline 09 & $\begin{array}{l}\text { Tengo más amigos en red, que conocí jugando un videojuego que } \\
\text { realizando otras actividades. }\end{array}$ & & & & \\
\hline 10 & $\begin{array}{l}\text { Mis amigos dicen que soy adicto a los videojuegos en red, yo pien- } \\
\text { so } \\
\text { Que se equivocan. }\end{array}$ & & & & \\
\hline 11 & $\begin{array}{l}\text { Mis padres me han llamado la atención porque paso más tiempo } \\
\text { Jugando en la red, que haciendo mis deberes. }\end{array}$ & & & & \\
\hline 12 & $\begin{array}{l}\text { Considero que estar más de tres horas jugando el internet no es } \\
\text { malo. }\end{array}$ & & & & \\
\hline 13 & $\begin{array}{l}\text { He gastado el poco dinero que invirtiendo en los beneficios que } \\
\text { ofrecen los videojuegos en red. }\end{array}$ & & & & \\
\hline 14 & Prefiero jugar videojuegos, que participar en reuniones familiares. & & & & \\
\hline 15 & $\begin{array}{l}\text { Me he olvidado de desayunar, almorzar y cenar por estar jugando } \\
\text { Videojuegos en red. }\end{array}$ & & & & \\
\hline 16 & $\begin{array}{l}\text { Suelo incitar a mis amigos para seguir jugando en red y poder ob- } \\
\text { tener más beneficios del juego. }\end{array}$ & & & & \\
\hline 17 & $\begin{array}{l}\text { Suelo estar mucho tiempo jugando en red que olvido comunicarme } \\
\text { con mi familia. }\end{array}$ & & & & \\
\hline 18 & Suelo llegar tarde a clases por estar jugando videojuegos en red. & & & & \\
\hline
\end{tabular}


19 Pienso que mis padres exageran al regañarme cuando me quedo jugando más de dos horas en el internet.

20 Prefiero invertir mi dinero para obtener beneficios del videojuego.

21 Cuando veo a mis amigos jugar siento la necesidad de jugar con ellos.

22 Prefiero jugar videojuegos que hacer deportes $u$ otra actividad.

23 Prefiero conversar mediante video chat mientras juego.

24 Pienso que debo jugar más tiempo para ganar más experiencia y subir a niveles más altos.

25 Suelo llegar tarde a mi trabajo y/o clases por quedarme un momento más jugando en red.

26 Tengo la necesidad de estar conectado en la red.

27 Me privo de cosas para ahorrar dinero y gastar en el internet.

28 Suelo a veces jugar en mis horas laborables si cuento con internet.

29 Mientras que el docente explica las clases yo estoy jugando algún videojuego.

30 Suelo perder la noción del tiempo cuando juego en red.

31 Prefiero pasar el tiempo jugando un videojuego a estar con amistades.

32 Descuido mis actividades por pensar o planear estrategias para ganar en los videojuegos en red.

33 Siento la necesidad de jugar un videojuego por internet a cada momento.

34 No cumplo con mis tareas porque me paso el tiempo jugando un videojuego.

35 Si soy adicto a los videojuegos, eso a la gente no debería importar.

36 Cuando no juego me pongo a ver a otras personas jugando

37 Suelo estar al pendiente de los niveles de juego en mis horas de Trabajo y/o en clases.

38 Mi familia ha tenido problemas porque eh estado mucho tiempo Jugando en el internet.

39 Cuando realizo otras actividades pienso constantemente en que estaría haciendo yo jugando videojuegos.

40 He sentido que la cantidad de horas de juego no son suficientes.

41 Suelo desvelarme jugando en la red toda una noche para ganar más niveles. Me siento ansioso por jugar en red y ganar más niveles.

\section{DISCUSIÓN}

La realización de la presente investigación tuvo como objetivo la construcción y validación de un instrumento que mida la adicción a los videojuegos en red. Por lo cual se elaboró el cuestionario construcción y propiedades psicométricas de un cuestionario para medir el nivel de adicción a videojuegos en red (CUAVIR), que consiste en una serie de ítems direccionados en el objetivo del constructo. De acuerdo con Cerda (1998), un cuestionario debe responder a dos requisitos básicos, el primero se refiere a la validez que debe existir en el constructo y el segundo es la fiabilidad.

El presente constructo cumple con estos requisitos demostrando tener una validez significativa y una 
buena fiabilidad según el alfa de Cronbach de 0.942 .

El cuestionario de adicción a videojuegos quedó compuesto por 42 ítems, distribuidos en las dimensiones Abstinencia, Resistencia, Interferencia de actividades, dependencia y conciencia de enfermedad. Con una buena consistencia interna mediante el coeficiente alfa de Cronbach de 0.942 , lo que indica una elevada fiabilidad.

En la primera etapa se realizó el estudio de validez de contenido por criterio de jueces utilizando el coeficiente $V$ de Aiken para la cuantificación de la misma, indicando que la prueba es muy confiable y su validez $(\mathrm{V}$ de Aiken $=0.89$ ) donde se muestra que es un instrumento que mide lo que verdaderamente pretende medir.

Los resultados presentados en nuestro estudio son favorables, pero es necesario reconocer ciertas limitaciones, pues una debilidad del cuestionario es la dimensión de conciencia de enfermedad, que está conformada por pocos ítems. Por ello, futuras investigaciones podrían ampliar el número de ítems en dicha dimensión.

\section{Declaración de financiamiento y de conflictos de interés:}

El estudio fue financiado por los autores, quienes declaran no tener conflictos de interés

\section{Correspondencia:}

Yeni Bellido Mamani

Correo electrónico:

yeni.bellido@upeu.edu.pe

Mónica Cahuana Cuti

Correo electrónico:

eliana.cahuana@upeu.edu.pe

\section{REFERENCIAS BIBLIOGRÁFICAS}

Carbonell, X., Guardiola, E., Beranuy, M. y Bellés, A. (2009). A bibliometric analysis of the scientific literature on Internet, video games, and cell phone addiction. Journal of The Medical Library Association, 97, 102-107. doi:10.3163/15365050.97.2.006

Carbonell, Guardiola, Beranuy y Bellés (2009). Cuestionario de experiencias relacionados a los videojuegos (CERV): Un instrumento para detectar el uso problemático de videojuegos en adolescentes españoles. Adicciones, 26 (4). Recuperado de https://www.adicciones.es/index. php/adicciones/

Carbonell (2019). El diagnóstico de adicción a videojuegos en el dsm-5 y la cie-11: retos y oportunidades para clínicos. Recuperado de https://doi.org/10.23923/pap.psicol2020.2935.

Choliz, M. \&Marco, C. (2010). Patrón de uso y dependencia de videojuegos en infancia y adolescencia. Anales de psicologia, 27(2). Recueprado de https://www.um.es/analesps/

Diario Correo, (2018). Estudio revela que el $45 \%$ de los videogamers en Perú son mujeres. Recuperado de https://diariocorreo.pe/tecnologia/estudiorevela-45-videogamers-peru-mujeres-804518/

Echeburúa E, Corral P. (2009). Adicción a las nuevas tecnologías y a las redes sociales. Pirámide. Recuperado de https://www.injuve.es/sites/ defaul/files/Revista98_completa_pdf

La Clasificación Internacional de Enfermedades incluirá los problemas asociados al juego digital. Recuperado de https://elpais.com/elpais/2017/12/21/ ciencia/1513852127_232573.html.

Escurra (1988). Cuantificación de la validez de contenido por criterio de jueces. Revista de psicología, 6 (1_2), 103-111. Recuperado de https://revista.pucp.edu.pe/index.php/psicologia/ article/view/455

Griffith, M (2005). Adicción a los videojuegos: Una revisión de la literatura. Behaviora Psychology / Psicología conductual (2005, enero) Vol. 13. Recuperado de https://www.researchgate.net/ publication/273951321_ADICCION_A_LOS_ VIDEOJEGOS_UNA_REVISION_DE_LA_ LITERATURA

Hernandez, Roberto; Fernández, Collado; Baptista, M. del P. (2014). Metodología de la Investigación. Recuperado de https://periodicooficial.jalisco.. gob.mx/sites/peridicooficial.jalisco.gob.mx/files/ metodologia_de_la_investigacion - Roberto_ hernandez_sampieri.pdf 
Huaraya (2013). Frecuencia de la adicción a los juegos en red y algunas de sus características clínico-epidemiologicas en jugadores de 14 y 19 años en cuatro cabinas de juegos de Arequipa 2013. 64 (2).

Luque y Degoy (2012) Adicciones tecnológicas: el docente como agente de prevención. Buenos aires. Recuperdo de https://www.aacademica. orp/000-072/289

Lemmens y Valkenburg (2009). Development and validation of game adiction scale for adolescents. Media Psychology, 12, 77-95. Recuperado de https://www.tandfonline.com
Montero y León, (2007). A guide for naming research studies in Psychology. International Journal of Clinicaland Health Psychology7. (3),847862. Recuperado de https://www.google.com/ url?sa=t\&source=web\&rct=j\&url=http: $w w w$. a e p c e s / i j c h p / G N E I P 07 es.pdf\&ved=2ahUKEwjM2NH-qdDrAhXjVkKHZE mDuQQFjABegQIBxAL\&usg=AOvVaw30WVBD metGxaOjoe7HVY3M\&cshid=1599251063340

Recibido: 09/07/2020

Aceptado: 02/10/2020 